hep-th/yymmnnn

\title{
Absence of resonant decay for metastable vacua in gauge theories of scalar fields
}

\author{
M. Cardella. S. Elitzur ${ }^{\dagger}$ and E. Rabinovic \\ Racah Institute of Physics, The Hebrew University of Jerusalem, 91904, Israel.
}

\begin{abstract}
We prove the impossibility of resonant decay of a metastable vacuum in a theory of a scalar field coupled to a gauge field. Our result extends to gauge theories of scalar fields a recent no go theorem for resonant tunneling in a pure scalar field theory.
\end{abstract}

PACS numbers:

\section{INTRODUCTION}

Understanding in detail the mobility patterns in the landscape is a challenging problem. In fact rather little is known about it and thus even a study of what may seem non generic paths is of interest. For example Tye [1], 2] has suggested to apply the phenomena of rapid tunneling [4], [5], 6], [7], [8] to shed a different light on the issue of the cosmological constant. Tunneling is generically heavily suppressed. In quantum mechanics it is known that there are special barrier configurations for which the suppression factor is removed and tunneling proceeds as if the barrier were transparent, this phenomenon is called

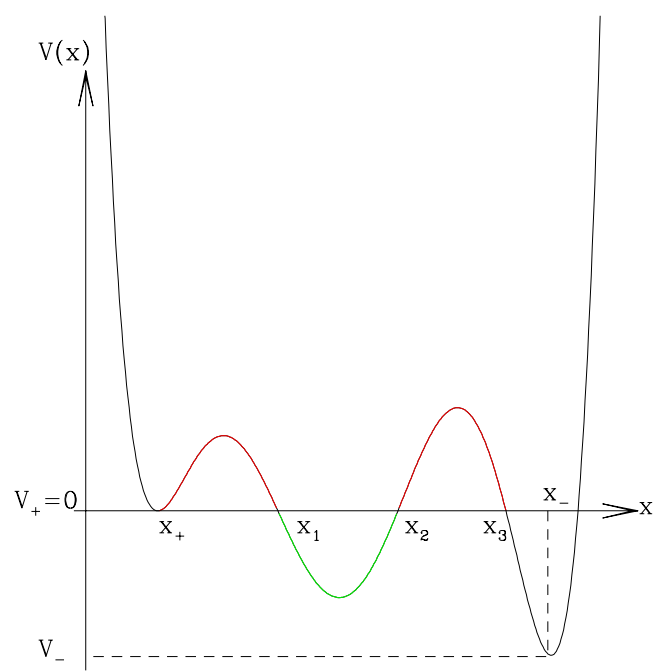

FIG. 1: Double barrier particle decay: In the semiclassical regime a metastable state with wave function localized around $x=x_{+}$decays to the true vacuum localized around $x=x_{-}$. If the length of the classically allowed region $x_{1}<x<x_{2}$ contains a half-integer number of de Broglie particle wavelength then quantum interference leads to resonant tunneling.

*Electronic address: matteo@phys.huji.ac.il

${ }^{\dagger}$ Electronic address: elitzur@vms.huji.ac.il

$\ddagger$ Electronic address: eliezer@vms.huji.ac.il resonant tunneling.

Although resonant tunneling is a well understood and observed phenomenon in quantum mechanics [9], [10], it does not easily extend to systems with an infinite number of degrees of freedom. Indeed a no-go theorem for resonant tunneling from a metastable vacuum in a scalar quantum field theory (SQFT) has been recently proved [4]. The aim of the present paper is to study whether theories with more structure then SQFT give a different outcome. Gauge theories had provided exits out of no-go theorems for pure scalar field theories. One example is the existence of solitons in $D>2$ in theories of a scalar field coupled to a gauge field, which are forbidden for a pure scalar field theory in $D>2$ by Derrick theorem 11]. Motivated by that, we will analyze the possibility of resonant tunneling in theories of scalar fields coupled to gauge fields with several metastable vacua. We will follow the ideas of the proof of the no-go theorem for SQFT [4], generalizing it to a gauge theory in any space-time dimension. The result is that in a theory of a scalar field coupled to a gauge field a homogenous metastable vacuum does not decay in a resonant fashion. This provides a no go theorem for resonant vacuum decay which generalizes [4] in allowing the presence of gauge field with generic coupling to the scalar field.

The organization of the paper is the following: In the next section we review resonant tunneling in quantum mechanics, leaving for the appendix the details of the derivations of the tunneling amplitudes. We then discuss what it would be required in order to have a similar phenomenon in quantum field theory, such as the constraints imposed by time-analytic continuation. In section III we generalize to a generic number of space-time dimensions $D$ the proof of the no go theorem of 4$]$ for resonant tunneling in SQFT, in order to be able to discuss resonant tunneling in gauge theories of scalar fields. In the appendix a derivation of quantum mechanical tunneling amplitudes and resonance conditions for multi-barrier potentials is reviewed.

\section{RESONANT TUNNELING: FROM QM TO QFT}

Quantum interference is typical in systems with a degenerate set of classical trajectories. In the double barrier potential $V(x)$ in figure 1, in the semiclassical regime a 


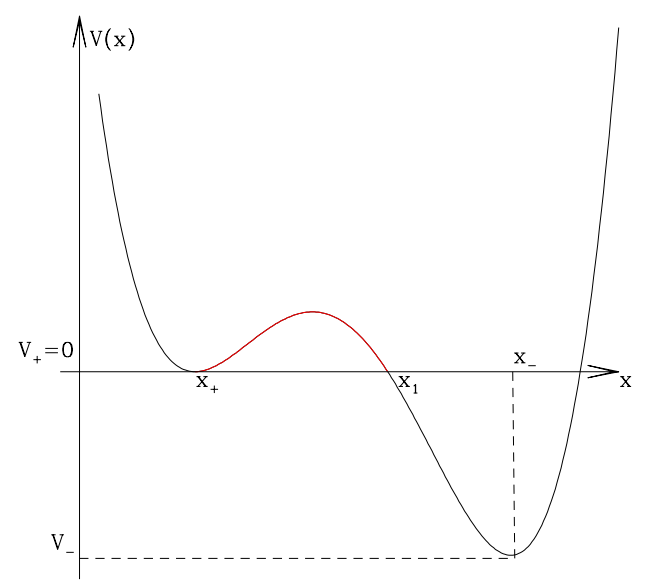

FIG. 2: In the semiclassical regime, a particle with wave function localized around a false vacuum $x=x_{+}$can decay to the true vacuum localized around $x=x_{-}$by quantum tunneling the barrier $x_{+}<x<x_{1}$.

point particle with wave function localized around the false vacuum $x=x_{+}$will decay to the true vacuum localized around $x=x_{-}$. The tunneling process involves an infinite number of decay paths, labeled by the number of oscillations in the classical allowed region $x_{1}<x<x_{2}$. Different oscillating paths can constructively interfere and, for specific particle wave lengths the double potential barrier becomes completely transparent. This phenomenon is called resonant tunneling, and it has been observed in various experiments [9],[10].

While the amplitude to decay by tunneling through the single barrier in fig. 2 is at leading order in $\hbar$ exponentially suppressed by a instanton action

$$
T=\frac{1}{\cosh \left(S_{I}\right)} \sim \exp \left(-\frac{1}{\hbar} \int_{x_{+}}^{x_{1}} d x \sqrt{2 V(x)}\right),
$$

in the double barrier case, if all the oscillating paths in region $x_{1}<x<x_{2}$ have the same phase at $x=x_{2}$, they create a constructive interference for the wave function entering the second forbidden region $x_{2}<x<x_{3}$. Constructive interference happens when the distance between the two inversion points $x=x_{1}$ and $x=x_{2}$ contains an half-integral number of de Broglie wave lengths

$$
S_{I I}=\frac{1}{\hbar} \int_{x_{1}}^{x_{2}} d x \sqrt{-2 V(x)}=\left(N+\frac{1}{2}\right) \pi
$$

for integers $N$. Equation (2) denotes a resonance condition, since in this case the amplitude to decay to $x=x_{-}$ reaches its maximum modulus

$$
\left|T_{+-}\right|=\frac{1}{\cosh \left(S_{I}-S_{I I I}\right)} .
$$

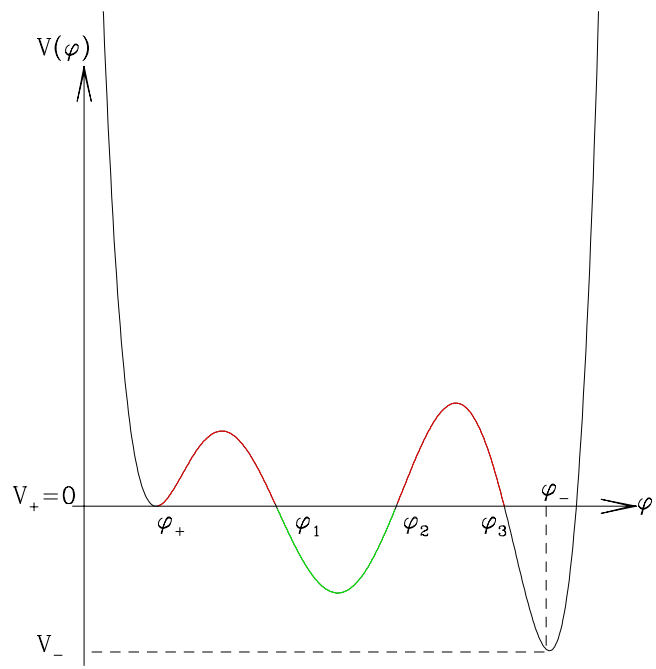

FIG. 3: Double barrier decay in QFT. The field in a false vacuum $\varphi=\varphi+$ can decay trough quantum tunneling toward lower energy density local minima of the effective potential.

$S_{I}$ and $S_{I I I}$ are the instanton actions that dominate the amplitude in the two forbidden regions $x_{+}<x<x_{1}$, and $x_{2}<x<x_{3}$,

$$
\begin{aligned}
S_{I} & =\frac{1}{\hbar} \int_{x_{+}}^{x_{1}} d x \sqrt{2 V(x)}, \\
S_{I I I} & =\frac{1}{\hbar} \int_{x_{2}}^{x_{3}} d x \sqrt{2 V(x)} .
\end{aligned}
$$

If $S_{I} \sim S_{I I I}$ the decay amplitude (3) gets close to one $\left|T_{+-}\right| \lesssim 1$, and thus the metastable state $x=x_{+}$becomes very short living. For this to happen it is required that $S_{I}-S_{I I I} \sim 0$, and that $S_{I I}$ satisfies eq.(2). In the semiclassical regime both $S_{I}$ and $S_{I I I}$ are large numbers in $\hbar$ units, thus the condition $S_{I}-S_{I I I} \sim 0$ requires a large amount of fine tuning for the shape of the potential $V(x)$.

In quantum field theory the post-tunneling dynamics would begin in a localized space-time region, in a way similar to the one-barrier tunneling field decay discussed in [12], [13]. The field tunnels in a finite space-time region by making a quantum jump from its original value $\varphi=\varphi_{+}$to the final vacuum value $\varphi=\varphi_{-}$, ( see figure 3 ). In the thin wall approximation, new and old vacua are separated by a domain wall, whose positive energy balances exactly the decrease in energy in the limited new vacuum region. The domain wall will finally expand by converting potential energy into wall kinetics energy.

The object that computes the amplitude per unit of space-time volume for the field to tunnel through a clas- 
sically forbidden region is the Euclidean path integral

$$
I=\int \mathcal{D} \varphi e^{-S_{E}[\varphi]}
$$

In the semiclassical approximation the dominant contributions to $I$ are Euclidean classical fields (instantons). These Euclidean fields satisfy a global constraint which is the Euclidean version of energy conservation

$$
\begin{aligned}
& \frac{\partial}{\partial t_{E}} \int d \vec{x}\left[\frac{1}{2}\left(\frac{\partial \varphi}{\partial t_{E}}\right)^{2}-\sum_{i=1}^{d} \frac{1}{2}\left(\frac{\partial \varphi}{\partial x_{i}}\right)^{2}-V(\varphi)\right] \\
= & \frac{\partial}{\partial t_{E}} \int d \vec{x}\left[\frac{1}{2}\left(\frac{\partial \varphi}{\partial t_{E}}\right)^{2}-U[\varphi]\right] \\
= & 0
\end{aligned}
$$

In quantum field theory (QFT) in order to have quantum interference a periodic field path $\varphi$, solution of the equations of motion with Lorentzian signature, would be required. Resonant tunneling would then follow if its action $S[\varphi]$ satisfied

$$
S[\varphi]=\left(N+\frac{1}{2}\right) \pi,
$$

for integer $N$.

This periodic path should be connected via time analytic continuation to Euclidean paths, the latter describing tunneling in classically forbidden regions of the potential. The question is whether in quantum field theory there are oscillating phenomena that parallel the oscillating particle paths in the region $x_{1}<x<x_{2}$ of figure 1.

In the classically allowed regions, where the potential for constant field configuration is less then the metastable vacuum energy density, the generalized potential $U[\varphi]$ of some classical field solution could become negative, (for example this could happen for the potential in fig. 3] if on a sufficiently large space region $\varphi$ assumes a value in the interval $\varphi_{1}<\varphi<\varphi_{2}$ ). If this happens one is forced to analytic continue the solution to real time $t_{E} \rightarrow i t_{E}=t$ at the point $\tilde{t}_{E}$ where $\partial_{t_{E}} \varphi\left(\tilde{t}_{E}, \vec{x}\right)=0$. Analytic continuation in $\tilde{t}_{E}=\tilde{t}$ requires $\partial_{t_{E}} \varphi\left(\tilde{t}_{E}, \vec{x}\right)=0=\partial_{t} \varphi(\tilde{t}, \vec{x})$.

In the following we will assume analytic continuation of an instanton $\varphi\left(t_{E}, \vec{x}\right)$ to Lorentzian time and then back to Euclidean time

$U[\varphi]\left(t_{E}^{1}\right)=U[\varphi]\left(t_{E}^{2}\right)=0, \quad U[\varphi]\left(t_{E}\right)<0, \quad t_{E}^{1}<t_{E}<t_{E}^{2}$.

The Lorentzian continuation $\varphi(t, \vec{x})$ is defined on the interval $\left[t_{1}, t_{2}\right]$, where $t_{1}=t_{E}^{1}$ and $t_{2}=t_{E}^{2}$.

Together with this solution there are equally dominant contributions from paths in which the Euclidean instanton is continued to a Lorentzian classical field $\varphi_{n}(t, \vec{x})$, which is a periodic extension of $\varphi$ for a time period $(2 n+1)\left(t_{2}-t_{1}\right)$, for integer $n . \varphi_{n}(t, \vec{x})$ would contribute to the path integral with action $S\left[\varphi_{n}\right]=$ $S[\varphi]_{(2 n+1)\left(t_{1}, t_{2}\right)}=(2 n+1) S[\varphi]_{\left(t_{1}, t_{2}\right)}$, where

$$
\begin{array}{r}
S[\varphi]_{\left(t_{1}, t_{2}\right)}= \\
\int_{t_{1}}^{t_{2}} d t \int d \vec{x}\left[\frac{1}{2}\left(\frac{\partial \varphi}{\partial t}\right)^{2}-\sum_{i=1}^{d} \frac{1}{2}\left(\frac{\partial \varphi}{\partial x_{i}}\right)^{2}-V(\varphi)\right]
\end{array}
$$

thus creating quantum interference in the path-integral.

A necessary condition for quantum interference in a multi-vacua potential to affect field decay is therefore the existence of a finite action classical Lorentzian solution $\varphi$ defined on a time interval $\left[t_{1}, t_{2}\right] . \varphi$ has vanishing time derivative $\partial_{t} \varphi\left(t_{1}, \vec{x}\right)=\partial_{t} \varphi\left(t_{2}, \vec{x}\right)=0$ in order to be analytically continued to instanton field solution. In the following, we will check whether $\varphi$ has non-vanishing action. We consider resonant decay in scalar fields theories, already discussed in [4], and the same issue in gauge theories of scalar fields, which is the focus of this paper.

\section{VACUUM DECAY IN A LANDSCAPE POTENTIAL AND THE QUESTION OF QUANTUM INTERFERENCE}

As discussed at the end of the previous section, in order to have quantum interference in vacuum decay the existence of a Lorentzian solution $\varphi(t, \vec{x})$ defined on a time interval $\left[t_{1}, t_{2}\right]$ with non-vanishing action satisfying eq. (7) is required. Analytic continuation of $\varphi$ to Euclidean signature in $t=t_{1}=t_{2}$ requires $\partial_{t} \varphi\left(t_{1}, \vec{x}\right)=\partial_{t} \varphi\left(t_{2}, \vec{x}\right)=0$. Moreover $\varphi$ must respect the total energy constraint. Since we are studying the decay of the false vacuum, $\varphi$ has to approach at spacial infinity the original metastable vacuum $\varphi_{+}$

$$
\lim _{|\vec{x}| \rightarrow \infty} \varphi(t, \vec{x})=\varphi_{+},
$$

and therefore

$$
\lim _{|\vec{x}| \rightarrow \infty} \partial_{\mu} \varphi(t, \vec{x})=0
$$

\section{A. No resonant tunneling in a scalar field theory}

Let us consider a scalar field theory in $D=1+d$ spacetime dimensions with Lagrangian

$$
\mathcal{L}=\frac{1}{2} \partial_{\mu} \varphi \partial^{\mu} \varphi-V(\varphi)
$$

where $V(\varphi)$ is a generic potential with various metastable vacua.

A necessary condition for the field $\varphi$ to have nonvanishing action in the interval $\left[t_{1}, t_{2}\right]$ is the strict positivity of the following functional 


$$
\mathcal{O}_{L}[\varphi]=\int_{t_{1}}^{t_{2}} d x_{0} \int d \vec{x}\left[\left(\partial_{0} \varphi\right)^{2}+\frac{1}{d} \sum_{i=1}^{d}\left(\partial_{i} \varphi\right)^{2}\right] .
$$

In fact, if $\mathcal{O}_{L}[\varphi]=0$, being an integral of a sum of positive terms, the derivatives of the field vanish identically and $S[\varphi]=S\left[\varphi_{+}\right]=0$.

By using

$$
\partial_{\mu} \varphi \partial_{\nu} \varphi=T_{\mu \nu}+\eta_{\mu \nu} \mathcal{L}
$$

$\mathcal{O}_{L}[\varphi]$ can be written in terms of the following combinations of diagonal components of the energy-momentum tensor $T_{\mu \nu}$

$$
\mathcal{O}_{L}[\varphi]=\int_{t_{1}}^{t_{2}} d x_{0} \int d \vec{x}\left[T_{00}+\frac{1}{d} \sum_{i=1}^{d} T_{i i}\right] .
$$

Energy-momentum conservation $\partial^{\mu} T_{\mu i}=0$ for the $i$ spacial component gives

$$
\begin{aligned}
\partial_{i} T_{i i} & =\sum_{j \neq i} \partial_{j} T_{j i}-\partial_{0} T_{0 i} \\
& =\sum_{j \neq i} \partial_{j}\left(\partial_{j} \varphi \partial_{i} \varphi\right)-\partial_{0}\left(\partial_{0} \varphi \partial_{i} \varphi\right) .
\end{aligned}
$$

This implies that

$$
\partial_{i} \int_{t_{1}}^{t_{2}} d x_{0} \int \prod_{j \neq i} d x_{j} T_{i i}=\partial_{i} I_{i i}=0
$$

the $T_{i i}$ density integrated over the space-time domain orthogonal to the $x_{i}$-direction and containing the time interval $\left(t_{1}, t_{2}\right)$ is constant along $x_{i}$. Therefore $I_{i i}$ can be computed at $x_{i}=\infty$, where it depends only on $V\left(\varphi_{+}\right)$, since all the field derivatives go to zero at infinity

$$
\begin{aligned}
I_{i i}= & \lim _{x_{i} \rightarrow \infty} \int_{t_{1}}^{t_{2}} d x_{0} \int \prod_{j \neq i} d x_{j} . \\
& \left(\frac{1}{2}\left(\partial_{0} \varphi\right)^{2}+\frac{1}{2}\left(\partial_{i} \varphi\right)^{2}-\frac{1}{2} \sum_{i=1}^{d}\left(\partial_{i} \varphi\right)^{2}-V(\varphi)\right) \\
= & -\int_{t_{1}}^{t_{2}} d x_{0} \int \prod_{j \neq i} d x_{j} V\left(\varphi_{+}\right) .
\end{aligned}
$$

On the other hand, $\partial^{\mu} T_{\mu 0}=0$ gives

$$
\partial_{0} T_{00}=\sum_{i=1}^{d} \partial_{i} T_{i 0}=\sum_{i=1}^{d} \partial_{i}\left(\partial_{i} \varphi \partial_{0} \varphi\right),
$$

that integrated all over the space gives energy conservation

$$
\partial_{0} I_{00}=\partial_{0} \int d \vec{x} T_{00}=0
$$

The energy equals the energy of our initial state at $t_{1}$. This being the final configuration of the Euclidean tunneling, its Euclidean energy is the same as the Euclidean energy of the original decaying state. Since both configurations have zero time derivatives, there is no distinction between Euclidean and Lorentzian energy. Assuming a homogeneous original false vacuum $\varphi=\varphi_{+}$,

$$
I_{00}=\int d \vec{x} V\left(\varphi_{+}\right) .
$$

By using eq.(17) and eq.(20) in the field functional $\mathcal{O}_{L}[\varphi]$ (12) one obtains

$$
\begin{aligned}
\mathcal{O}_{L}[\varphi] & =\int_{t_{1}}^{t_{2}} d x_{0} I_{00}+\frac{1}{d} \sum_{i=1}^{d} \int d x_{i} I_{i i} \\
& =\int_{t_{1}}^{t_{2}} d x_{0} \int d \vec{x}\left[V\left(\varphi_{+}\right)-\frac{1}{d} \sum_{i=1}^{d} V\left(\varphi_{+}\right)\right] \\
& =0 .
\end{aligned}
$$

which shows that $\partial_{0} \varphi=|\vec{\nabla} \varphi|=0$ in the time interval $\left[t_{1}, t_{2}\right]$, thus implying that the field $\varphi$ has zero action in the time interval $\left[t_{1}, t_{2}\right]$. Moreover, since the field is constant in $\left[t_{1}, t_{2}\right]$ it must be equal to the false vacuum $\varphi=\varphi_{+}$. This shows that an intermediate analytic continuation to Lorentzian signature is impossible, and the full tunneling event is described by instanton fields in Euclidean signature. Thus one concludes that for the decaying of a metastable vacuum in a pure scalar field theory with a multi-vacua potential there is no quantum interference in the semiclassical approximation. This proves the no go theorem for resonant tunneling in a pure scalar field theory in a generic number of space-time dimensions $D$. Generalization of this result to an arbitrary number of interacting scalar fields is straightforward, since also in this case one can compute each of the terms in the spacelike diagonal components of the energy-momentum tensor appearing in (14) by going at spacial infinity, where they all equals minus the energy of the metastable vacuum.

Recently, 7] have shown that special non-homogenous field configurations with finite energy with respect to a metastable vacuum do exhibit resonant decay. These states circumvent the no-go theorem of [4] in allowing a non-vanishing r.h.s. for eq.(20), which makes (21) strictly positive, thus allowing for oscillating in time field solutions.

It is also interesting to observe that instantons describing motion in forbidden regions are non constant fields connected by analytic continuation to two real time solutions, the field before and after tunneling, at point with vanishing Euclidean time derivative. Instantons are therefore the Euclidean version of the oscillating field forbidden by the no-go theorem. The no-go theorem must fail in Euclidean signature, and it does. The Euclidean 
version of eq.(12) is

$$
\mathcal{O}_{E}[\varphi]=\int_{t_{E}^{1}}^{t_{E}^{2}} d x_{0} \int d \vec{x}\left[\left(\partial_{0} \varphi\right)^{2}+\sum_{i=1}^{d}\left(\partial_{i} \varphi\right)^{2}\right] .
$$

This functional can be written in terms of the Euclidean stress tensor $T_{\mu \nu}^{E}$ by summing over the diagonal components of

$$
\partial_{\mu} \varphi \partial_{\nu} \varphi=T_{\mu \nu}^{E}+\delta_{\mu \nu} \mathcal{L}_{E}
$$

where the Euclidean Lagrangian is

$$
\mathcal{L}_{E}=\frac{1}{2} \partial_{\mu} \varphi \partial_{\mu} \varphi+V(\varphi)
$$

By using eq. (23) and the Euclidean conservation law $\partial_{\mu} T_{\mu \nu}^{E}=0$, one can rewrite (22) as

$$
\mathcal{O}_{E}[\varphi]=-2 \frac{D}{D-2} \int_{t_{E}^{1}}^{t_{E}^{2}} d t_{E} \int d \vec{x} V\left(\varphi\left(t_{E}, \vec{x}\right)\right) .
$$

The above equation makes sense only when the integral in the r.h.s. is non-negative. This is achieved when there is a large enough region of Euclidean space where the field acquires values in classically allowed regions where $V(\varphi)<0$, such as the regions $\varphi_{1}<\varphi<\varphi_{2}$ and $\varphi>\varphi_{3}$ for the potential in figure (3).

\section{B. No quantum interference in the decay of a scalar field coupled to a gauge field}

We study now whether a theory of a scalar field coupled to a gauge field admits non-constant classical field solutions of Lorentzian time signature that can be analiticaly continued to Euclidean signature in two separate occasions.

We consider the Lagrangian

$$
\mathcal{L}=-\frac{1}{4} F_{\mu \nu}^{a} F^{a \mu \nu}+\frac{1}{2} D_{\mu} \varphi\left(D^{\mu} \varphi\right)^{\dagger}-V(\varphi)
$$

In the semiclassical approximation the Euclidean path integral is dominated by Euclidean classical fields. These fields satisfy the global constraint

$$
\begin{gathered}
\partial_{0} \int d \vec{x}\left[\frac{1}{2} \sum_{i=1}^{d}\left(F_{0 i}^{a}\right)^{2}+\frac{1}{2} D_{0} \varphi\left(D_{0} \varphi\right)^{\dagger}\right. \\
\left.-\frac{1}{4} \sum_{i, j=1}^{d}\left(F_{i j}^{a}\right)^{2}-\sum_{i=1}^{d} D_{i} \varphi\left(D_{i} \varphi\right)^{\dagger}-V(\varphi)\right]=0,
\end{gathered}
$$

which is an Euclidean version of energy conservation. We choose the original metastable state energy equal to zero
$V\left(\varphi_{+}\right)=0$ so that the previous equation gives

$$
\begin{aligned}
& \int d \vec{x}\left[\frac{1}{2} \sum_{i=1}^{d}\left(F_{0 i}^{a}\right)^{2}+\frac{1}{2} D_{0} \varphi\left(D_{0} \varphi\right)^{\dagger}\right] \\
= & \int d \vec{x}\left[\frac{1}{4} \sum_{i, j=1}^{d}\left(F_{i j}^{a}\right)^{2}+\sum_{i=1}^{d} D_{i} \varphi\left(D_{i} \varphi\right)^{\dagger}+V(\varphi)\right] \\
= & : U\left[\varphi, A_{\mu}^{a}\right] .
\end{aligned}
$$

Given the Euclidean fields $\varphi\left(t_{E}, \vec{x}\right), A_{\mu}^{a}\left(t_{E}, \vec{x}\right)$, (27) is an equation for $t_{E}$. For values of the Euclidean fields in regions where $V(\varphi)<0$, the r.h.s of eq.(27) might become negative $U\left[\varphi, A_{\mu}^{a}\right]<0$. We assume that this is the case on an interval $t_{E}^{1}<t_{E}<t_{E}^{2}$, and check the possibility of a analytic continuation of the fields $\varphi\left(t_{E}, \vec{x}\right)$ and $A_{\mu}^{a}\left(t_{E}, \vec{x}\right)$ to Lorentzian signature $t_{E} \rightarrow i t_{E}=t$ at $t_{E}^{1}=t_{1}$ and back to Euclidean signature $t \rightarrow-i t=t_{E}$ at $t_{E}^{2}=t_{2}$. The Lorentzian classical solutions $\varphi(t, \vec{x})$ and $A_{\mu}^{a}(t, \vec{x})$ are then defined on the interval $\left[t_{1}, t_{2}\right]$, they dominate the path integral and have both vanishing time derivative at $t=t_{1}$ and $t=t_{2}$, as required by analytic continuation. In order to check if there is a degeneracy of Lorentzian motions that can produce interference in the path integral we will check if $\varphi(t, \vec{x})$ and $A_{\mu}^{a}(t, \vec{x})$ have a non vanishing action in the interval $\left[t_{1}, t_{2}\right]$.

To this aim we again use the energy-momentum tensor

$$
T_{\mu \nu}=F_{\mu}^{\rho a} F_{\rho \nu}^{a}+D_{\mu} \varphi\left(D_{\nu} \varphi\right)^{\dagger}-\eta_{\mu \nu} \mathcal{L}
$$

and consider the following sum over the diagonal components of $T_{\mu \nu}$

$$
\begin{aligned}
& \mathcal{O}_{L}\left[\varphi, A_{\mu}^{a}\right]= \\
& \quad \int_{t_{1}}^{t_{2}} d x_{0} \int d \vec{x}\left[D_{0} \varphi\left(D_{0} \varphi\right)^{\dagger}+\frac{1}{d} \sum_{i=1}^{d} D_{i} \varphi\left(D_{i} \varphi\right)^{\dagger}\right] \\
& +\int_{t_{1}}^{t_{2}} d x_{0} \int d \vec{x}\left[\frac{d-1}{d} \sum_{i=1}^{d}\left(F_{0 i}^{a}\right)^{2}+\frac{1}{d} \sum_{i, j=1}^{d}\left(F_{i j}^{a}\right)^{2}\right] \\
& =\int_{t_{1}}^{t_{2}} d x_{0} \int d \vec{x}\left[T_{00}+\frac{1}{d} \sum_{i=1}^{d} T_{i i}\right] .
\end{aligned}
$$

$\mathcal{O}_{L}\left[\varphi, A_{\mu}^{a}\right] \geq 0$ is a semi-definite positive functional, and a field solution $\left(\tilde{\varphi}, \tilde{A}_{\mu}^{a}\right)$ with a non-vanishing action gives $\mathcal{O}_{L}\left[\tilde{\varphi}, \tilde{A}_{\mu}^{a}\right]>0$. We use the component equations of $\partial^{\mu} T_{\mu \nu}=0$ integrated over space-time subregions, in order to show that indeed for every classical solution $\mathcal{O}_{L}\left[\varphi, A_{\mu}^{a}\right]=0$. This implies that every solution defined on a finite time interval $\left[t_{1}, t_{2}\right]$ which can be analiticaly continued to Euclidean signature has to be a constant field configuration equal to the false vacuum. The conclusion will be that field paths describing the full tunneling event are purely Euclidean and therefore there is no resonant decay. 
The overall $T_{00}$ space integral is set to be equal to the total false vacuum energy $V\left(\varphi_{+}\right)=0$ that is our initial condition in time, therefore the space integral of $T_{00}$ vanishes in the r.h.s of (29).

On the other hand, the component equation $\partial^{\mu} T_{\mu i}=0$ gives

$$
\begin{aligned}
\partial_{i} T_{i i} & =\partial_{0} T_{0 i}-\sum_{j \neq i} \partial_{j} T_{j i} \\
& =\partial_{0}\left(F_{0}^{\rho a} F_{\rho i}^{a}+D_{0} \varphi\left(D_{i} \varphi\right)^{\dagger}\right) \\
& -\sum_{j \neq i} \partial_{j}\left(F_{i}^{\rho a} F_{\rho j}^{a}+D_{i} \varphi\left(D_{j} \varphi\right)^{\dagger}\right) .
\end{aligned}
$$

By integrating the previous equation over a space-time volume orthogonal to the $x_{i}$ direction one gets

$$
\begin{aligned}
& \quad \partial_{i} \int_{t_{1}}^{t_{2}} d x_{0} \int \prod_{j \neq i} d x_{j} T_{i i}= \\
& \quad \int \prod_{j \neq i} d x_{j} \int_{t_{1}}^{t_{2}} d x_{0} \partial_{0}\left(-\sum_{j \neq i} F_{0 j}^{a} F_{j i}^{a}+D_{0} \varphi\left(D_{i} \varphi\right)^{\dagger}\right) \\
& -\int_{t_{1}}^{t_{2}} d x_{0} \int \prod_{j \neq i} d x_{j} \sum_{j \neq i} \partial_{j}\left(F_{i}^{\rho a} F_{\rho j}^{a}+D_{i} \varphi\left(D_{j} \varphi\right)^{\dagger}\right) \\
& =0 .
\end{aligned}
$$

The previous equation states that the above integral is constant along $x_{i}$, therefore it is equal to its value for $x_{i} \rightarrow \infty$, where it easier to see that it vanishes, since all the field derivatives vanish at spacial infinity, and the potential goes to the false vacuum $V\left(\varphi_{+}\right)=0$.

We have shown that the r.h.s of eq. (29) vanishes, from the same equation one can read that the two Lorentzian continuations $\varphi(t, \vec{x}), A_{\mu}^{a}(t, \vec{x})$ have a vanishing action (26) in the interval $\left[t_{1}, t_{2}\right]$. We conclude that there is no quantum interference in vacuum decay in a theory of a scalar field coupled to a gauge field. Therefore even in the presence of a gauge field a metastable vacuum cannot decay in a resonant fashion. Generalization of the above result to a gauge theory of an arbitrary number of interacting scalar fields is straightforward, since all the steps of the proof we gave go through also in this case.

\section{CONCLUSIONS}

We studied whether in a theory of a scalar field coupled to a gauge field a homogeneous metastable vacuum can decay in a resonant fashion. The answer turns out to be negative and thus our result generalizes the no go theorem for resonant tunneling in a pure scalar field theory [4]. The proof follows the line of [4], appropriately generalized to arbitrary space-time dimensions and to the presence of a gauge field. Gauge interaction have exhibited in the past novel non-perturbative phenomena such as the existence of solitons in $D>2$, forbidden for a pure scalar field theory by Derrick theorem [11]. Yet, in the present case they did not come to rescue, and resonant tunneling from a homogeneous false vacuum and its interesting implications for the dynamics of the landscape mentioned in the introduction are also forbidden for theories of scalar fields coupled to gauge fields.

The authors of the no go theorem for resonant tunneling in a pure scalar field theory [4] have recently proposed a way to circumvent their own no-go theorem [7]. They consider the decay of inhomogenous exited states of a metastable vacuum, rather then the decay of the false vacuum itself. These states represent quite ad hoc non-homogeneous initial configurations of the scalar field, and whether they can emerge as the outcome of a previous tunneling event in the landscape it remains to be explained. Besides the need to supply a natural mechanism that makes the bubbles of [7] to contract, one should also estimate the occurrence of such events in the landscape. This would be an essential ingredient in order to determine whether the proposal of [7] represents an actual possibility for rapid vacuum decay. As stressed in [7], the present no go theorem does not apply to non homogeneous initial states. Notice that if indeed non homogeneous initial states can have resonant tunneling, solitonic solutions present in gauge theories may naturally supply such initial states as ground states in given topological sectors.

\section{APPENDIX: WAVE MECHANICS METHODS FOR TUNNELING AMPLITUDES THROUGH MULTIPLE BARRIERS}

\section{A. Tunneling through one barrier potential}

Let us consider the tunneling problem represented in figure 4, the semiclassical wave function for a particle of unit mass with energy $E$ in region $\mathrm{I}$ is given by

$\Psi_{I}(x)=\alpha_{I}^{R} \frac{e^{i S_{0}(x) / \hbar}}{(2(E-V(x)))^{1 / 4}}+\alpha_{I}^{L} \frac{e^{-i S_{0}(x) / \hbar}}{(2(E-V(x)))^{1 / 4}}$,

where

$$
S_{0}(x)=\int_{x_{+}}^{x} d x \sqrt{2(E-V(x))}
$$

is the reduced action.

In order to compute the tunneling probability one has to compare the semiclassical wave functions in region I with that one in region III, beyond the barrier. Semiclassical approximation breaks down near the classical turning point $x=x_{+}$, and in fact (32) is divergent there. The two expressions for the semiclassical wave functions outside the barrier (region I) and inside the barrier (region II), can be obtained by solving the Schroedinger equation near $x=x_{+}$, with the linearized potential 


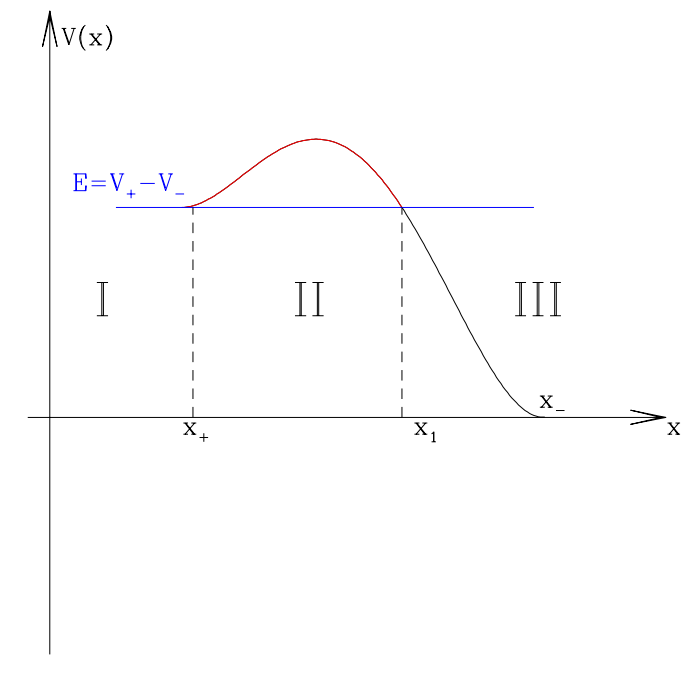

FIG. 4: A particle in region I with energy $E=V_{+}-V_{-}$has a non vanishing probability to tunnel to region III through the classically forbidden potential barrier of region II

$2(E-V(x)) \sim 2 F_{0}\left(x-x_{+}\right)$. Semiclassical approximation is valid on the region

$$
\left|x-x_{+}\right|>>\left(\frac{\hbar^{2}}{2 F_{0}}\right)^{1 / 3}
$$

(see for example 14]), therefore by comparing the two asymptotic expansion of the solution on the left and on the right of the turning point $x=x_{+}$one can obtain a relation between the semiclassical wave functions in the two regions, called connection formula.

It is amusing to see how through a simple method one can recover the same connection formulae without the knowledge of the solution of the Schroedinger equation for the linearized potential near the inversion point. Starting with the semiclassical wave function (32) in the allowed region I, one can obtain the correct expression for the semiclassical wave function beyond the turning point in region II by circumventing $x=x_{+}$in the complex plane along a semicircular path centered in $x=x_{+}$: $x-x_{+} \rightarrow e^{i \varphi}\left(x-x_{+}\right)$. There are two ways to circumvent the turning point, a clockwise path $\varphi=-\pi$ and the anti-clockwise $\varphi=\pi$ one, each way producing a different wave function inside the barrier. It turns out that the correct wave function inside the barrier is given by summing the two contributions:

$$
\begin{aligned}
\Psi_{I I}(x)= & e^{-\Theta}\left(\alpha_{I}^{R} e^{-i \pi / 4}+\alpha_{I}^{L} e^{i \pi / 4}\right) \frac{e^{-S_{0}^{\prime}(x) / \hbar}}{(2(V(x)-E))^{1 / 4}} \\
& +e^{\Theta}\left(\alpha_{I}^{R} e^{i \pi / 4}+\alpha_{I}^{L} e^{-i \pi / 4}\right) \frac{e^{S_{0}^{\prime}(x) / \hbar}}{(2(V(x)-E))^{1 / 4}}
\end{aligned}
$$

where

$$
S_{0}^{\prime}(x)=\int_{x_{1}}^{x} d x \sqrt{2(V(x)-E)},
$$

and

$$
\Theta=\frac{1}{\hbar} \int_{x_{+}}^{x_{1}} d x \sqrt{2(V(x)-E)} .
$$

By using $\Psi^{I I}$ given by eq. (35), one can reach region III by circumventing on the complex plane the turning point $x=x_{2}$ with two semicircular paths centered in $x=x_{2}$. The wave function in region III $x>x_{2}$ outside the barrier is then given by

$$
\Psi_{I I I}(x)=\alpha_{I I I}^{R} \frac{e^{i S_{0}^{\prime \prime}(x) / \hbar}}{(2(E-V(x)))^{1 / 4}}+\alpha_{I I I}^{L} \frac{e^{-i S_{0}^{\prime \prime}(x) / \hbar}}{(2(E-V(x)))^{1 / 4}},
$$

with

$$
S_{0}^{\prime \prime}(x)=\frac{1}{\hbar} \int_{x_{1}}^{x} d x \sqrt{2(E-V(x))} .
$$

The relation between the wave function coefficients in region I and III can be cast in the following nice formula

$$
\begin{aligned}
\left(\begin{array}{c}
\alpha_{I I I}^{R} \\
\alpha_{I I I}^{L}
\end{array}\right) & =\left(\begin{array}{cc}
\cosh \Theta & -i \sinh \Theta \\
i \sinh \Theta & \cosh \Theta
\end{array}\right)\left(\begin{array}{c}
\alpha_{I}^{R} \\
\alpha_{I}^{L}
\end{array}\right) \\
& =\mathcal{R}_{\Theta} \cdot\left(\begin{array}{c}
\alpha_{I}^{R} \\
\alpha_{I}^{L}
\end{array}\right)
\end{aligned}
$$

which shows that the wave function which enters from region I into the forbidden region II, it reappears in region III as "rotated" by the matrix $\mathcal{R}_{\Theta}$

$$
\mathcal{R}_{\Theta}=\left(\begin{array}{cc}
\cosh \Theta & -i \sinh \Theta \\
i \sinh \Theta & \cosh \Theta
\end{array}\right) .
$$

The factor $i$ in the above "rotation" matrix ensures that its determinant is one, a necessary condition to preserve the wave function norm.

The probability for transmission through a single barrier is given by

$$
|T|^{2}=\left|\frac{\alpha_{I I I}^{R}}{\alpha_{I}^{R}}\right|^{2}=\frac{1}{(\cosh \Theta)^{2}},
$$

and in the semiclassical approximation that we are considering $\Theta>>1$

$$
\begin{aligned}
|T|^{2} & =\frac{1}{(\cosh \Theta)^{2}} \sim 4 e^{-2 \Theta} \\
& =4 \exp \left(-\frac{2}{\hbar} \int_{x_{1}}^{x_{2}} d x \sqrt{2(V(x)-E)}\right) .
\end{aligned}
$$

This last formula reproduces the tunneling probability given in eq. (1). 


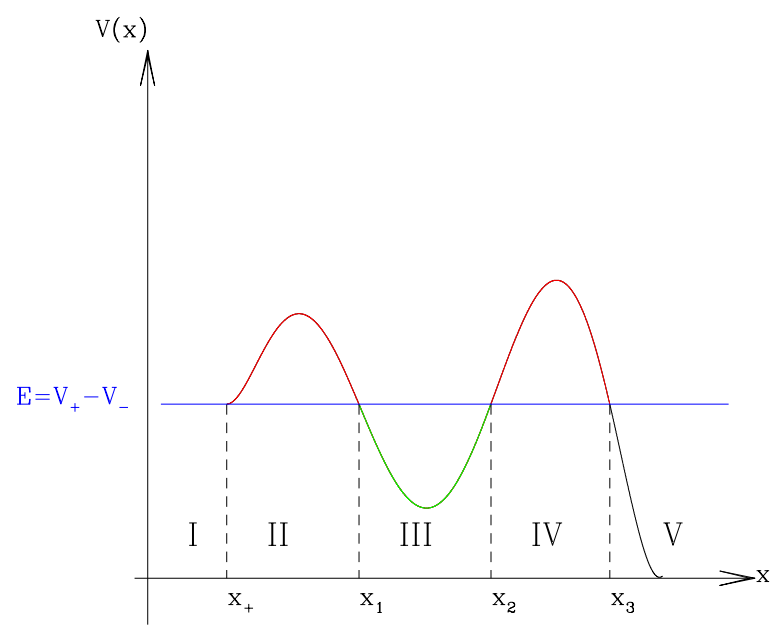

FIG. 5: Double barrier potential: Due to quantum interference there is a finite spectrum of energies such that the probability to tunnel through the double barrier is higher than the probability to tunnel through a single barrier. This is due to the presence of the intermediate classically allowed region III, where the various oscillating paths between the classical turning points $x=x_{1}, x=x_{2}$ can quantum interfere in a constructive way, giving rise to resonant tunneling.

\section{B. Tunneling through a double barrier potential}

For a double barrier tunneling (fig. 5) one can compute the full "rotation" matrix which rotates the wave function of region I into the wave function of region $\mathrm{V}$

$$
\vec{\alpha}_{\text {out }}=\mathcal{R}_{\Theta_{1}} \mathcal{R}_{\delta} \mathcal{R}_{\Theta_{2}} \vec{\alpha}_{\text {in }},
$$

where $\mathcal{R}_{\Theta_{1}} \mathcal{R}_{\delta} \mathcal{R}_{\Theta_{2}}$ is given by and

$$
\Theta_{2}=\frac{1}{\hbar} \int_{x_{2}}^{x_{3}} d x \sqrt{2(E-V(x))}
$$

are the corresponding (hyperbolic) angles in the classically forbidden regions.

The transmission coefficient for double barrier tunneling is obtained by $T=\alpha_{\text {out }}^{R} / \alpha_{i n}^{R}$, with the condition $\alpha_{\text {out }}^{L}=0$.

Computation gives the tunneling probability

$$
|T|^{2}=\frac{1}{\cos ^{2} \delta \cosh ^{2}\left(\Theta_{1}+\Theta_{2}\right)+\sin ^{2} \delta \cosh ^{2}\left(\Theta_{1}-\Theta_{2}\right)} .
$$

The term in $\cos \delta$ corresponds to the product of the decay probabilities, it is the non-resonant contribution, dominant in the limit $\delta=0$, when interference in the intermediate region plays no role. For $\delta=0$ the two hyperbolic angles $\Theta_{1}$ and $\Theta_{2}$ sum together giving the sum of the rotations. The term in $\sin \delta$ is the effect of the quantum interference, and it depends on the difference between $\Theta_{1}$ and $\Theta_{2}$.

The tunneling probability can be closed to one in the resonant condition

$$
\delta=(n+1 / 2) \pi, \quad \Theta_{1} \sim \Theta_{2} .
$$

Notice that $\Theta_{1} \sim \Theta_{2}$ requires a large amount of fine tuning, since in the semiclassical regime that we are considering both $\Theta_{1}$ and $\Theta_{2}$ are large numbers in $\hbar$ units.

\section{Tunneling through $N$ barriers}

Given $N$ barriers separated by $N-1$ classically allowed regions, the matrix which connects the wave functions on the two regions extending to infinity is given by $\mathcal{R}_{N}=$ $\prod_{k=1}^{N} \mathcal{R}_{\Theta_{k}} \mathcal{R}_{\delta_{k}}$

$$
\mathcal{R}_{N}=\prod_{k=1}^{N}\left(\begin{array}{cc}
\cosh \Theta_{k} & -i \sinh \Theta_{k} \\
i \sinh \Theta_{k} & \cosh \Theta_{k}
\end{array}\right)\left(\begin{array}{cc}
e^{i \delta_{K}} & 0 \\
0 & e^{-i \delta_{K}}
\end{array}\right),
$$

$$
=\cos \delta\left(\begin{array}{cc}
\cosh \left(\Theta_{1}+\Theta_{2}\right) & -i \sinh \left(\Theta_{1}+\Theta_{2}\right) \\
i \sinh \left(\Theta_{1}+\Theta_{2}\right) & \cosh \left(\Theta_{1}+\Theta_{2}\right)
\end{array}\right) \quad \text { where }
$$$$
+i \sin \delta\left(\begin{array}{cc}
\cosh \left(\Theta_{1}-\Theta_{2}\right) & -i \sinh \left(\Theta_{1}-\Theta_{2}\right) \\
i \sinh \left(\Theta_{1}-\Theta_{2}\right) & \cosh \left(\Theta_{1}-\Theta_{2}\right)
\end{array}\right) \text {. }
$$

$$
\begin{aligned}
& \Theta_{k}=\frac{1}{\hbar} \int d x \sqrt{2 V(x)}, \\
& \delta_{k}=\frac{1}{\hbar} \int d x \sqrt{-2 V(x)} .
\end{aligned}
$$

There may be various resonant tunneling conditions, the simplest one corresponds to the existence of a energy bound state common to all the $N-1$ classical allowed regions. If the incoming particle has this bound state energy the total tunneling probability turns out to be

$$
\begin{gathered}
\delta=\frac{1}{\hbar} \int_{x_{1}}^{x_{2}} d x \sqrt{2(E-V(x))} \\
\Theta_{1}=\frac{1}{\hbar} \int_{x_{+}}^{x_{1}} d x \sqrt{2(E-V(x))},
\end{gathered}
$$
and is the interference phase responsible for resonant tunneling, while 


$$
|T|^{2}=\frac{1}{\cosh ^{2}\left(-\sum_{k=1}^{N}(-)^{k} \Theta_{k}\right)},
$$

and resonant tunneling occurs for

$$
\sum_{k=1}^{N}(-)^{k} \Theta_{k}=0 .
$$

This resonance condition extends to a generic number of barriers $N$ the resonance condition

$$
\Theta_{1}-\Theta_{2}=0
$$

which has been obtained for the double barrier potential in figure 5 .

\section{ACKNOWLEDGMENTS}

M.C. would like to thank A. Schwimmer for stimulating discussions. The work of M.C. was supported by Superstring Marie Curie Training Network under the contract MRTN-CT-2004-512194. The work of S.E. was partially supported by the Israel Science Foundation, the Einstein Center in the Hebrew University, and by a grant of DIP (H.52). The work of E.R. was partially supported by the European Union Marie Curie RTN network under contract MRTN-CT-2004-512194, the AmericanIsrael Bi-National Science Foundation, the Israel Science Foundation, The Einstein Center in the Hebrew University, and by a grant of DIP (H.52).
[1] S. H. Tye, "A new view of the cosmic landscape," arXiv:hep-th/0611148.

[2] S. H. Tye, "A Renormalization Group Approach to the Cosmological Constant Problem," arXiv:0708.4374 [hepth].

[3] S. R. Coleman and F. De Luccia, "Gravitational Effects On And Of Vacuum Decay," Phys. Rev. D 21, 3305 (1980).

[4] E. J. Copeland, A. Padilla and P. M. Saffin, "No resonant tunneling in quantum field theory," arXiv:0709.0261 [hep-th].

[5] S. Sarangi, G. Shiu and B. Shlaer, "Rapid Tunneling and Percolation in the Landscape," arXiv:0708.4375 [hep-th].

[6] A. R. Brown, S. Sarangi, B. Shlaer and A. Weltman, "A Wrinkle in Coleman - De Luccia," Phys. Rev. Lett. 99, 161601 (2007) arXiv:0706.0485 [hep-th]].

[7] P. M. Saffin, A. Padilla and E. J. Copeland, "Decay of an inhomogeneous state via resonant tunnelling," arXiv:0804.3801 [hep-th].
[8] Q. G. Huang and S. H. Tye, "The Cosmological Constant Problem and Inflation in the String Landscape," arXiv:0803.0663 [hep-th].

[9] L.Chang, L. Esaki and R. Tsu, Appl. Phys. Lett. 24 (1974) 593.

[10] H. Mizuta and T. Tanoue The Physics and Applications of Resonant Tunneling Diodes, Cambridge University Press, Cambrbridge (1995).

[11] G.H. Derrick, J. Math. Phys. 5, 1252 (1964)

[12] S. R. Coleman, "The Fate Of The False Vacuum. 1. Semiclassical Theory," Phys. Rev. D 15, 2929 (1977) [Erratum-ibid. D 16, 1248 (1977)].

[13] C. G. Callan and S. R. Coleman, "The Fate Of The False Vacuum. 2. First Quantum Corrections," Phys. Rev. D 16, 1762 (1977).

[14] E. Merzbacher, Chapter 7, Quantum Mechanics, 2nd Edition, John Wiley, 1970. 